\title{
Shaping Pro-Social Interaction in VR
}

\author{
An Emerging Design Framework
}

\author{
Joshua McVeigh-Schultz \\ Computational Media \\ UC Santa Cruz \\ Santa Cruz, CA, USA \\ jmcveigh@ucsc.edu
}

\author{
Anya Kolesnichenko \\ Computational Media \\ UC Santa Cruz \\ Santa Cruz, CA, USA \\ akolesni@ucsc.edu
}

\author{
Katherine Isbister \\ Computational Media \\ UC Santa Cruz \\ Santa Cruz, CA, USA \\ kisbiste@ucsc.edu
}

\begin{abstract}
Commercial social VR applications represent a diverse and evolving ecology with competing models of what it means to be social in VR. Drawing from expert interviews, this paper examines how the creators of different social VR applications think about how their platforms frame, support, shape, or constrain social interaction. The study covers a range of applications including: Rec Room, High Fidelity, VRChat, Mozilla Hubs, Altspace VR, AnyLand, and Facebook Spaces. We contextualize design choices underlying these applications, with particular attention paid to the ways that industry experts perceive, and seek to shape, the relationship between user experiences and design choices. We underscore considerations related to: (1) aesthetics of place (2) embodied affordances, (3) social mechanics, (4) and tactics for shaping social norms and mitigating harassment. Drawing on this analysis, we discuss the stakes of these choices, suggest future research directions, and propose an emerging design framework for shaping pro-social behavior in VR.
\end{abstract}

\section{CCS CONCEPTS}

- Human-centered computing $\rightarrow$ HCI design and evaluation methods; Human-centered computing $\rightarrow$ HCI theory, concepts and models; Human-centered computing $\rightarrow$ Interactive systems and tools

KEYWORDS: Virtual reality, social VR, pro-social interaction

ACM Reference format:

Joshua McVeigh-Schultz, Anya Kolesnichenko, Katherine Isbister. 2019. Shaping Pro-Social Interaction in VR: An Emerging Design Framework. In 2019 CHI Conference on Human Factors in Computing Systems Proceedings (CHI 2019), May 4-9, 2019, Glagsow, Scotland, UK. ACM, New York, NY, USA. Paper 564, 12 Pages. https://doi.org/10.1145/3290605.3300794

Permission to make digital or hard copies of all or part of this work for personal or classroom use is granted without fee provided that copies are not made or distributed for profit or commercial advantage and that copies bear this notice and the full citation on the first page. Copyrights for components of this work owned by others than the author(s) must be honored. Abstracting with credit is permitted. To copy otherwise, or republish, to post on servers or to redistribute to lists, requires prior specific permission and/or a fee. Request permissions from permissions@acm.org).

CHI 2019, May 4-9, 2019, Glasgow, Scotland, UK.

(๑) 2019 Copyright is held by the owner/author(s). Publication rights licensed to ACM. ACM ISBN 978-1-4503-5970-2/19/05...\$15.00.

DOI https://doi.org/10.1145/3290605.3300794

\section{INTRODUCTION}

Social virtual reality (VR) represents a growing set of multiuser applications that enable people to interact with one another in virtual space through VR head-mounted displays (HMDs). Commercially available applications include: VRChat, Rec Room, AltspaceVR, High Fidelity, Facebook Spaces, Anyland, and Mozilla Hubs (to name just a few). As part of a diverse and rapidly evolving media ecology, social VR applications vary widely terms of purpose, aesthetics, theme, functionality, interaction mechanics, and emergent social norms [26, 31]. Accordingly, each application reflects a different answer to the question, "what does it mean to 'be social' in VR?" [26]. Like traditional 2D social media forms on the web, different platforms privilege different dimensions of sociality and reflect different design approaches to social interaction. New fundamental questions arise, however, from the kinds of embodied and spatialized experiences that immersive presence in VR affords. For example, aspects of embodied presence magnify the experience of harassment in social VR $[1,26]$. And what may at first appear to be minor differences in mechanics can have a profound impact on the kinds of social interactions that emerge in a social VR environment.

In this paper, we seek to elucidate the constellation of design choices that shape pro-social interactions in commercial social VR. To address this goal, we conducted in-depth interviews with designers, developers, and other leading experts involved in the creation of commercial social VR applications. Drawing from our interviewees' first-hand knowledge of this emerging area of design practice, we mapped seven social VR applications in terms of the different design approaches that underlie social interaction in the respective applications. While these interviews focused on creators rather than users of social $\mathrm{VR}$, the creators' perceptions of user experiences was nevertheless a salient topic, and where possible, we sought to clarify the kinds of user data and empirical evidence that served to ground the experts' claims. From this research, we propose a preliminary design framework to shed light on a 
range of design choices that shape pro-social interaction in social VR.

With the anticipated growth of the social VR sector, this research represents a timely opportunity to study the relationship between design choices and social practices associated with virtual co-presence. Finally, we aim to clarify the stakes of these choices, and to outline research questions for future empirical fieldwork with users as well as design research in laboratory contexts.

\subsection{Background}

Research on multi-user VR is informed by a long history of research on collaborative virtual environments (CVEs) [1-3, $6,9,36,37]$, ethnographies of virtual worlds [4, 5, 23, 30, 40], and related accounts of social activity in virtual environments $[7,8,12,14,22,28,29,34]$. But as the commercial social VR sector grows and evolves, new fundamental questions arise about the kinds of embodied and spatialized experiences that immersive presence in VR affords. For instance, popular attention has recently pointed to the ways that embodied presence in social VR can magnify conflict or harassment [27, 35], underscoring the importance of designing for social safety in shared immersive environments. Likewise, researchers have an opportunity to understand the ways that design decisions can support positive aspects of social interaction in VR, for instance by carefully managing aspects of personal space [42] or social proxemics [4,5].

The rise of commercially available VR has driven a new research agenda in HCI [38] and directed questions of multiuser interaction in VR towards a new set of real-world and experimental contexts, including: collaborative learning [41], medical training [33], dance [39], and hybrid physical-virtual interaction [16]. In addition, analysis of game mechanics in VR has significantly bridged insights from industry and academic research. These include a comprehensive set of guidelines for usability and playability in VR [11] as well as more popular media accounts from the VR design community, for example [17]. Aside from a few preliminary studies $[26,31]$ however, less has been done, to draw design lessons about social interaction from commercial VR platforms. For example, how do affordances of personal space [42] and social proxemics [4,5] relate to issues of harassment in these platforms?

The emerging design knowledge of the social VR industry is an important data point for HCI researchers to capture and analyze. In particular, there is a need to bridge design knowledge about pro-social interaction that is otherwise sequestered or siloed within competing companies. Our interviewees expressed a "rising tide raises all boats" type of shared interest in mitigating negative social experiences in social VR, since a negative experience in one application might make someone less likely to want to explore social VR in other applications. Industry design knowledge may be tacit or incomplete, but it fills in important gaps in academic research, since industry design experience reflects real world engagement with design choices and grapples with the complex ways that user practices evolve over time. This approach enables us to ask different sorts of questions. What are the key factors, trade-offs, and priorities in prosocial VR design? Where are experts in this field aligned and where are they divergent on these topics? And how do differences within industry approaches map onto the range of social contexts that social VR applications support?

\subsection{Preliminary Work}

We conducted preliminary research using an autobiographical landscape analysis approach to map a slice of the social VR topography [26]. Drawing on these preliminary findings, we developed some initial hypotheses about the right factors for supporting pro-social interaction in social VR. These factors formed the topic areas of the interviews we conducted for this study.

\subsection{Fieldsites}

Rec Room was released in June of 2016. Modeled on a "rec center from 1987," Rec Room emphasizes playing games such as paint ball, co-op adventures, and 3D charades. In the Rec Center area, participants can manipulate a variety of objects and engage in activities like ping-pong, and basketball. From this main public area, they can travel to other more crafted game experiences. Avatars in Rec Room have simple humanoid features. Rec Room collects user data from a combination of analytics (for example tracking how often user creations are adopted by others) with deep community involvement through frequent in-world Q\&A sessions, and feedback/bug reports via a Discord forum.

AltspaceVR - released in May of 2015 and later acquired by Microsoft in October of 2017-supports an eclectic combination of experiences including chatting with others, playing games, and attending live events. AltspaceVR was touted since its founding for its accessibility to a wide range of devices including Gear VR. New visitors initially appear as robot avatars but can customize their avatars once registered. AltspaceVR collects user data from a combination of analytics (return rate, duration of use, etc.) along with deep community engagement through monthly town halls and a large moderator team that frequently draws qualitative insights from interactions with users. 
Facebook Spaces, released in April of 2017, focuses on supporting virtual interactions between people who are already friends on Facebook. Interactions are localized around a shared round table, and participants can "go" to different place by activating spherical 360 backgrounds. Facebook Spaces also sets itself apart by encouraging participants to choose avatars based on their Facebook photos. Users of Facebook Spaces are unlikely to encounter strangers since they can only interact with people they have already friended on Facebook. During its inception, the Facebook Spaces product team conducted fieldwork studying how young people hang out in physical space. Later during development, they conducted user testing with pairs of friends.

VRChat opened to Steam in February of 2017 and was soon after flooded with new users. Known for its "wild west" ethos, VRChat has at times struggled with harassment but has also created a unique home for wideranging forms of creative expression, with participants taking on personas and inventing new social rituals and memes. The application supports custom worlds and avatars as well as games like Capture the Flag and Battle Discs. For this study, we interviewed a 3rd party content creator who drew insights from her personal interactions with VRChat users for whom she designs.

Mozilla Hubs is a lightweight social VR meeting space tool for webVR. The project was created by a team largely composed of developers formerly of AltspaceVR. Released by Mozilla on April 26, 2018, Hubs are device agnostic, and users can join using a range of hardware options (including Vive, Oculus Rift, Gear VR, Cardboard, and desktop). Hubs' options for meeting spaces include an office environment, a medieval castle area, a rock-face mountain vista, and custom environments users can create through a browser interface. Users can bring in media from the web including images and 3D models. By design, Mozilla Hubs does not collect data from its users, so the development team's knowledge of user experience draws from a combination of internal testing and engagement with users through community events and forums.

Anyland, released on Steam in October of 2016, is the creation of co-founders and developers Scott Lowe and Philipp Lenssen. Unlike other customizable social VR environments, Anyland does not allow importing of 3D models. Instead, all environments, objects, avatars and interactions have been authored from scratch by a small community of committed users who have built more than one thousand unique 'areas.' By design, the world has a fairly simple graphical look, although some users create fairly sophisticated looking materials by importing $2 \mathrm{D}$ images to create custom textures. Anyland's developers communicate directly with users ("we visit them in Anyland and they tell us what they're doing") and closely follow a user forum that can be accessed from within Anyland.

High Fidelity VR is a platform whose founders include Philip Rosedale, CEO and founder of Linden Labs (maker of Second Life). Released as an open beta in April of 2016, High Fidelity underscores in-world construction tools with an emphasis on building your own world to inhabit. This ethos of a world-in-construction extends to the infrastructure of High Fidelity itself, and the platforms' developers work in-world in full view of visitors. More recently High Fidelity has experimented with large-scale public events and performances, bringing hundreds of users together into the same virtual space. High Fidelity collects user data through in-house and external user testing along with forum feedback related to bugs and new feature requests.

\section{METHODS}

Our team brings together background in design research, social science, as well as experience in designing for social VR. We modeled this study on similar design research that draws from expert interviews to develop a framework or set of design guidelines $[18,20]$.

\subsection{Interviews}

We interviewed eleven subjects with ties to social VR applications (Rec Room, AltspaceVR, High Fidelity, Anyland, Mozilla Hubs, and VRChat). These included six women, five men, and one self-described "cyborg" from a range of locations in North America and Europe. They represented a range of work roles including: founder, platform developer, content creator, avatar designer, community support coordinator, strategist, and product manager. Given their status as industry experts, most of our interviewees agreed to be explicitly identified by their names in this study.

\subsubsection{Recruiting}

Our recruitment efforts combined outreach to our own professional networks, social media, and local events. For two applications, VRChat and Facebook Spaces, we were unable to find representatives willing to participate directly, possibly due to the onerousness of getting a release approved or due to discomfort on with sharing proprietary details. In the case of Facebook Spaces, we instead drew 
insights from public presentations and articles written by key product designers. In the case of VRChat, we were able to interview a $3^{\text {rd }}$ party content creator who designed models, environments, and avatars for VRChat users.

\subsubsection{Interview Procedures}

Interviews lasted for 1.5-2 hours. Some were individual and others were group interviews. Interviews took place in private areas within the respective VR environments. We decided from the outset that it would be important to conduct our interviews inside the environments that we were studying, in order to ground the conversation with concrete examples. For instance, when discussing a particular avatar affordance, menu option, or environment, we could directly interact with the features being discussed. We conducted the interviews as a remote pair, with one of us participating in VR and another serving as a moderator and note taker who followed the events in $2 \mathrm{D}$ via screen share and guiding new question topic areas. We recorded interviews via OBS screen/audio capture. Interviews were semi-structured, and questions covered a range of topics including: place and space, context cues, methods of shaping and enforcing social norms (such as moderation), embodied affordances like locomotion and personal space, avatar systems, and social mechanics of blocking and friending. These interview topics were drawn from categories surfaced by exploratory research [26]. Finally at the end of the interview we shared our preliminary design framework to gather their input on what might be missing and how to prioritize the various factors.

\subsubsection{Interview Analysis}

To analyze our interviews, we conducted qualitative coding of interviews to surface insights about design approaches and thematic clusters. We utilized a semi-automated transcription and video annotation tool (Temi), and we organized responses and notes into a master spreadsheet. For analysis we took cues from Saldaña's approach to qualitative coding [32]. We began by organizing responses according to major topic areas, then added secondary concept annotation, and finally organized annotations into emergent themes and clusters. For responses that relied on embodied cues in VR, we returned to the video to gather additional context. The first author took on the role of code bookkeeper. Our first round of coding cleaved somewhat closely to the interview question categories, but since interviewee responses reshaped our topics of interest, the resulting codes shifted our initial categories to more closely reflect interviewee concerns. We then performed a secondary coding exercise to identify particular strategies for achieving design goals and clustered interview responses around areas of commonality and difference. As a final coding pass we identified a high level grouping of design perspectives according to the following social VR categories: (A) open world environments with heavy human involvement in moderation and culture-formation. (B) open world environments without heavy moderation (characterized by either free-for-all or strong localized user control over their own areas). (C) closed environments where people get together with those they already know from "real" life.

\section{FINDINGS}

In the following section we unpack the design approaches elucidated by our expert interviewees, attending to areas of convergence, diversity, and anomaly. Especially among the large open platforms where a user is likely to encounter strangers, we found convergence around broad design themes involving the role of place and space, community engagement, moderation, social catalysts and activity structures, social mechanics of friending/muting/blocking etc., and other embodied affordances including a range of communication modalities. However, we also observed fairly substantial divergence in terms of particular mechanics underlying these broad design areas. Furthermore, for non-open-world platforms such as Facebook Spaces and Mozilla Hubs, we noted less focus on supporting safety and security, and more investment in design goals associated with: supporting device interoperability, empowering a range of participation modalities, and bridging social encounters in VR with the outside world.

\subsection{Place and Space as Social Frame}

All of our interviewees described the important role that aesthetics and architectural features of place play in scaffolding social interaction. Indeed, places in social VR are often designed to leverage the social expectations of related environments from the physical world.

\subsubsection{Aesthetics of place shape expectations and behavior}

A designer who creates avatars and custom environments for VRChat users (through a $3^{\text {rd }}$ party company) commented that she tries to leverage people's expectations about familiar places. She describes the physiological impact place associations in VR as a kind of "shadow." "You bring your life into VR with you. That means that if you've ever been somewhere and you go to a place in VR that is like that you get a shadow of that feeling-a weak version of that feeling." For example, her tropical cove [Fig. 1] conjures up feelings of being on the beach for those who have those associations. "That's the strength of a familiar." 


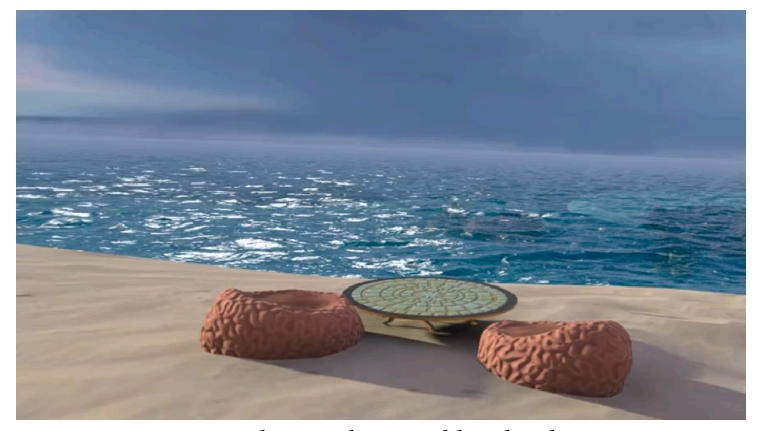

Figure 1: A tropical cove designed by third-party content creator for users of VRChat

Developer Cory Nolan described the world of Rec Room as themed on a prototypical rec center from the year 1987. With this approach in mind, she described their design approach as "family friendly" with bright colors, rounded edges that target a "general appeal." Rec Room's community support coordinator, Tamara Hughes, noted that certain places can prompt specific social cues by leveraging social expectations from their "real life" analogues. Describing an auditorium environment that they use for $Q$ \& A with players, she noted "this space didn't actually used to have the seating and levels and it used to be a madhouse sometimes, because people would just [stand] at the front. Then once we put the seating in, they saw it as a theater type space, and they [understood] that in real life you would sit down and be quiet." Similarly, Rec Room's designers eliminated a "locker room" environment due to its association with "locker room talk." Elaborating on this perspective, Nolan notes that "rooms are behavior."

Lenssen of Anyland, echoed this sentiment, noting that a place's aesthetic is indicative of the kinds of social encounters users are likely to have there. "If you go visit an area that has your kind of crowd, it's almost like in the real world where you would go to a certain bar. If you maybe want to meet some people, or if you want some more relaxed, chilled atmosphere, maybe you go to a cafe, or if you want some privacy, you stay at home. It's kind of a very fluid system, so to speak, in the real world where you don't have to friend or unfriend people all the time [as you go about your day]. You actually just go to the kind of location where you want certain social activity to take place and that's what we're trying to go for with Anyland."

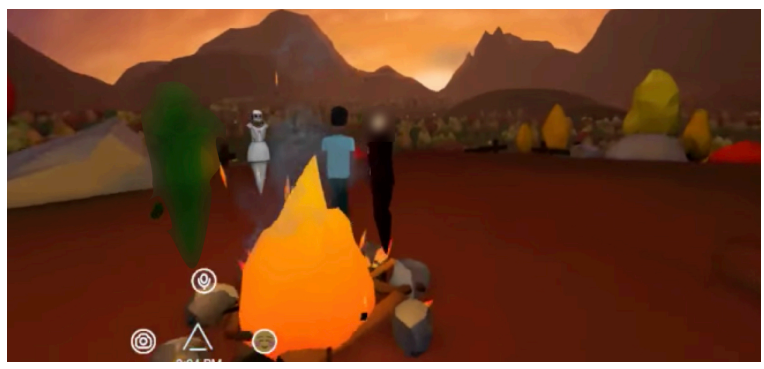

Figure 2: Campfire in AltspaceVR

\subsubsection{Architecture of Spaces}

Several designers described the importance of architectural features in the environment to support a sense of safety. For example, a content creator for VRChat users noted that for environments outside, providing cover with an enclosure makes people feel more secure. Similarly, Evan Sforza, a designer for AltspaceVR, remarked on how architectural features like cantilever overhangs provide a sense of protection against threats from above.

The impact of layout on geometries of social interaction also appeared in several examples. Facebook Spaces' product designers settled on a circular table to emphasize the shared eyelines necessary for a conversation [10]. And AltspaceVR's Sforza similarly emphasized the importance of circular gathering spots as conversational anchors. Kapur echoed this perspective in her description of the "spirit of the campfire" as a place where you come together with and share stories, share anecdotes, and have fun" [Fig. 2]. Rec Room developers and community managers also stressed the importance of architectural layout in an auditorium room (with a stage for speakers and seats for audience members) that was designed for Q\&A sessions between the player community and the Rec Room developers. More fantastical "architectural" features such as a giant bear, nonplayer character (NPC) that looks at whoever has the microphone also orient people to the social ritual of the $\mathrm{Q} \&$ A.

\subsubsection{Custom Environments}

All of the applications we examined were also either offering, or planned to soon offer, features that enabled users to create their own custom environments. For some, like Rec Room, this development was more recent and reflected a shift towards offering users more agency in shaping the aesthetics and social expectations of custom rooms. Perhaps the most extreme example of this design approach is represented by the application Anyland, in which everything can be created in-world including the user's own avatar-they begin as a ghostly empty body [Fig. 
3]. In addition to crafting their own avatars, Lenssen noted that in Anyland "everybody creates their own areas."

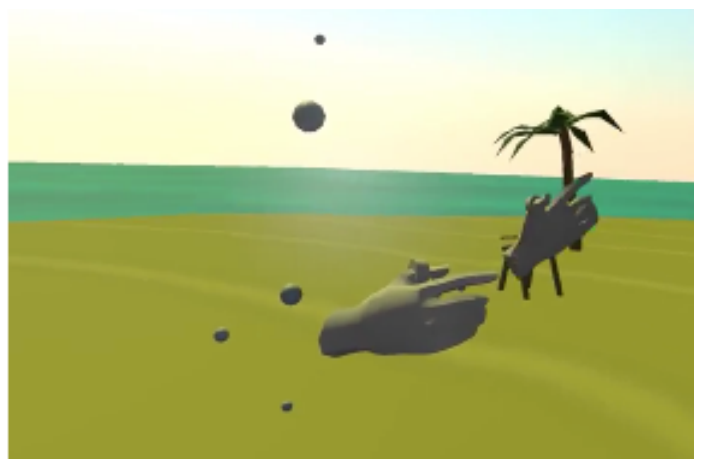

Figure 3: New user in Anyland with only hands and a "ghost" body, standing on a beach.

\subsection{Shaping Social Norms}

While all of the industry experts we spoke to expressed an interest in having a positive influence on the culture of their user communities, they demonstrated a fair degree of diversity in their design approaches to the question of how to shape social norms.

\subsubsection{Social Catalysts}

A community support coordinate for Rec Room, Adam Dormier, noted the importance of "stuff laying around that you can fool with" which serve as "social lubricants" in the Rec Center, such as a ping pong, dodge balls, basketballs, and traffic cones. AltspaceVR also creates social catalysts that they describe as environmental "ice breakers." Ishita Kapur, senior product manager of AltspaceVR, noted "we have icebreaker activities associated with the campfire, so "things like a marshmallow [roasting]. You can grill burgers for each other, and we have a little firecrackers that you can put in the fire and have them explode. [We wanted to] encourage that spirit of fun [and these icebreaker objects are a] productive way for people to kind of play in the space." Despite the appeal of these sorts of catalysts, on a more cautionary note, a content creator for VRChat users noted that objects like guns and knives can also function as negative social catalysts, encouraging harassment.

\subsubsection{Activities to Focus Social Engagement}

Social VR applications also support pro-social interaction by focusing shared attention on more structured activities such as games or creation tasks. Rec Room's Dormier noted that such activities offer "a shared context... like paintball, dodgeball, laser tag," etc. In the case of Mozilla Hubs and Facebook Spaces, both applications foreground conversation as a core activity. Facebook Spaces also connects to Facebook Live and Messenger, so that VR participants can converse with those outside of VR.

AltspaceVR initially placed less emphasis on foregrounding specific activities over others. Instead, it has positioned itself more as a platform that hosts content other people create. Sforza of AltspaceVR notes "it was never really our focus to as a team go and build an activity. And we were really hoping that SDK developers would be the ones to go and build activities.... We never really sat down and said, how do we design a great activity ourselves?" Kapur echoes this sentiment noting that "[We want] AltspaceVR is to be something that the community can make their own." Activities include VR dance parties, games like Holograms Against Humanity, Ted-style professional talks, karaoke nights, LGBT meetups, and even a wedding. "We hope that you can always find something that speaks to you and if not, we try to make it as easy as possible for people to create their own events, create their own activities.

In Anyland, activities are similarly up to users to craft, but Anyland's developers have designed Anyland's interface to foreground activities of in-world content creation and traveling to areas that others have created. Lenssen explained that Anyland offers "casual creation tool in VR, so you don't need to take off the headset and go into Blender or 3D programs right here without any knowledge, so that's a big part of the activities.... [Or] you can just click on the Friends dialog or the Areas dialogue and basically use this as a chat universe, where you can just go to areas that you like and chat with people. Or you can visit game areas, or horror areas, or... PvP shooting games... or gardens, where you can grow flowers and stuff like that." Lenssen commented that they "try to be not biased towards any activity."

\subsubsection{Community Engagement and Moderation}

A significant subset of the interviewees emphasized community outreach as a key element of supporting prosocial interaction in VR. Rec Room's Nolan underscored inclusivity and a desire to make people of different ages, nationalities, genders, sexual orientations, as well as those with disabilities feel equally welcome. Dormier noted that the initial community of Rec Room created the seeds of a positive community. "We started off with kind of a good nucleus for a community of folks that were just excited about the hardware and we're just generally kind people... so we started to grow a community of people who are respectful of this sort of social mission that we had, and everything we set forth in the code of conduct. So as we've 
grown, we... have maybe leaned on... that community to help encourage new people to abide by those social norms."

For High Fidelity, the seeds of positive community are shaped by the role played by greeter - in world employees of High Fidelity who answer questions and guide new users at key locations. Alexia Mandeville, a UX designer at High Fidelity noted that, for many first-time users, "a lot of people just have a conversation with the greeters and ask what High Fidelity is about... and the greeters usually walk them through domains they can go to, how they should interact with them. Greeters are our representatives."

Kapur described AltspaceVR's community ethos as the "spirit of the campfire." "A lot of people when they first go to campfire, the fact that there's always a moderator to kind of show them the ropes and kind of get the conversation going and make sure they feel grounded and make sure they feel at home." One of the ways that AltspaceVR maintains this sense of community is by empowering power users as "ambassadors and people that we would send out into the community to go and just continue to spread positivity and spread teamwork throughout." Kapur: "The fact that the community is empowered to kind of just make the space their own, has meant we've been able to lean on a few community members that serve as ambassadors as well."

Rec Room empowers individual users in a different way-by enabling custom room owners to police their own environments. If "rooms are behavior" as Nolan noted earlier, "this means if you set the room to public, it is now considered the same thing as if you're behaving in public. If you have a room that's sexually explicit, you're kind of in charge of policing the content of that room. And if other people are coming in and making toxic stuff in your room, we're going to moderate you, as if that was you who did it. So you want to always be careful about what you allow and don't allow in your rooms because it essentially has to reflect on you. And we kind of just set that as a guideline."

VRChat enables private rooms as well but has a more laissez faire attitude to moderation. A content creator for VRChat users noted that "VRChat is like a wild west, where you're on your own completely.... When [an area] says "open" on VRChat, you can't really stop people from being awful. The only thing you can do is to find your own friends circle and hang with them." From this creator's perspective, private environments in VRChat serve as a respite from the "wildness" of public areas.

Anyland has less resources for moderation but similarly relies on users policing their own custom areas. "Our focus is to give the area editors the tools to sort of maintain order within that area. For example, you can, as an area owner, kick somebody [out] if you want to or you can keep them [out] for 5 minutes or something like that. And you can also block people from joining the area if you disagree with their style or something." This approach also enables Anyland to be agnostic about what kind of behavior is appropriate in any given area, since according to Lenssen, "what might be appropriate in one area is not necessarily appropriate in another area.”

\subsubsection{Incentive Systems}

Lenssen of Anyland described discomfort with certain kinds of incentive systems that could be corrosive to community. For example, scoring systems can foster competition and envy. Despite a somewhat laissez faire stance, Lenssen admits he leans towards incentivizing "peaceful and friendly and constructive community." Lenssen considers these aspects of structural incentives to have a foundational impact with a host of cascading social implications. For example, by letting content-creators see scores for how often their content is reused by others, "they create a community that is constructive, friendly, that wants to share." In this way, incentive systems can lay the groundwork for either positive or negative forms of social contagion.

\subsection{Embodiment and Social Mechanics}

Despite some notable exceptions, we observed a general convergence with respect to design choices related to embodiment and social mechanics. These include features involving avatar affordances, locomotion features like teleportation, personal space bubbles, and social mechanics of friending and blocking. The specifics of implementation however reveal meaningful differences.

\subsubsection{Communicative affordances and social mechanics}

A number of applications have developed communication features that support pro-social interaction in novel ways that extend beyond the familiar affordances of the human body. For instance, in Rec Room, there are voice indicators above avatars to help you understand who is speaking at a given time. Rec Room avatars also cycle through various emotional expressions. Hughes commented that facial expressions "are more weighted towards the happy side than the frown side, which I think is part of what makes Rec Room such a friendly place, because everyone looks happy all the time." Such social augmentations begin to stretch the repertoire of face-to-face expression. And alternative communication modalities, such as "life-size" 
emojis, make this "stretching" even more explicit. Kapur noted that in AltspaceVR, "using emojis in some ways as substitutes for gestures, like a hand raise, or hand claps" especially when audiences are engaging with speakers, such as in town hall events. In other cases, new kinds of embodied communication rituals are instigated by users. For instance, a content creator for VRChat users noted the importance of gestures like reciprocal head patting or virtual "feeding" as an important way of communicating affection, since hugs are a less satisfying social ritual in VR due to a lack of physical feedback.

Handshake gestures came up among several of our interviewees as an area of some complexity since in many of the platforms the handshake doubles as a friending mechanism. Unintended friending along with the social pressure to friend can be quite problematic. Friending is also complicated due to the function of real time virtual presence combined with the expectation of reciprocity, which together makes for a socially awkward combination. Sforza of AltspaceVR remarked how in the physical world, "it's not like you get someone's number, and then you're following them around waiting for them to respond to you. You text them, and then you separate, and go your separate ways, and maybe they get back to you, maybe they don't. Maybe it makes sense to have some kind of temporal separation between the actual requests, and when things are really considered as a crystallization of a friendship."

While AltspaceVR doesn't codify this kind of temporal delay, Sforza noted that "we don't actually tell you, whether or not they accepted.... We don't want to put that pressure on the person right then to either accept or decline. We wanted to give them the freedom by not drawing any attention to what choice the person made in that moment to the other person who requested it, it would just create this awkward tension." Otherwise "[we'd be] reducing the human complexity to this very rudimentary, simple, simplistic form of expression.”

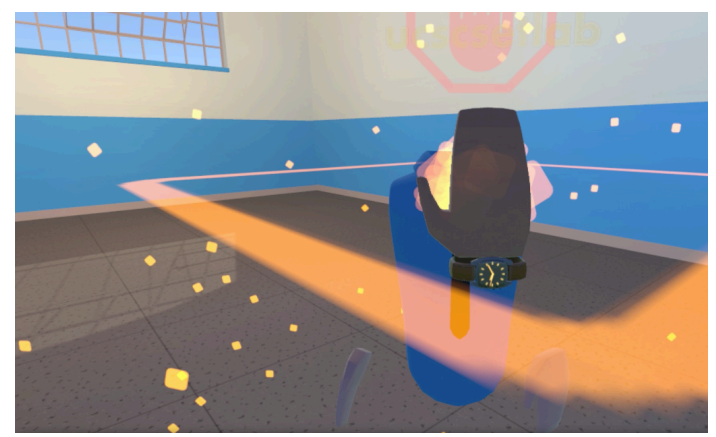

Figure 4: Rec Room's “Talk to the hand” gesture for

\subsubsection{Embodied Actions for Preventing Harassment}

Nearly all of the applications we examined utilized tools for preventing harassment that a user could activate in the moment. These included blocking/reporting, muting, a "panic button" (in the case of Anyland), as well as personal bubbles (which were often turned off by default). For most of the applications, blocking occurs when a user point at the offending party and pulls up a moderation menu to select "block." Some applications also collapse "blocking" and "reporting," thus forcing users to select a reporting category before they can complete the block action.

Rec Room has a strikingly user-friendly variation on this blocking mechanic. In Rec Room, users can simple hold their hands up to the harasser with a "talk to the hand" gesture (a kind of embodied skeuomorphism). An advantage of this "talk to the hand" gesture is that victimswho may be flustered during a harassment incident-do not have to go through the cumbersome process of pointing at the person and selecting a menu option before they are able to block the offender.

One challenge of blocking actions that require the victim orient to the offender is that harassers often attempt to game this mechanic by escaping quickly. This issue can be resolved however by several applications, including Anyland and Rec Room, which offer a menu option for "recent" users that the victim has interacted with.

Another broad category of user-directed moderation is the personal space bubble. When the personal space bubble is activated and another user gets to close, their avatar will begin to disappear at the overlap. Nearly all of the applications we examined employed some form of personal space management in this way.

Rec Room's personal space management also interacts with its teleportation system in subtle ways that gracefully handle the proxemics of personal space when multiple users attempt to teleport into the same area. In particular, when a user attempts to teleport into another user's personal space bubble, they instead will see their dashed teleport preview line turn from green to red [Fig. 5].

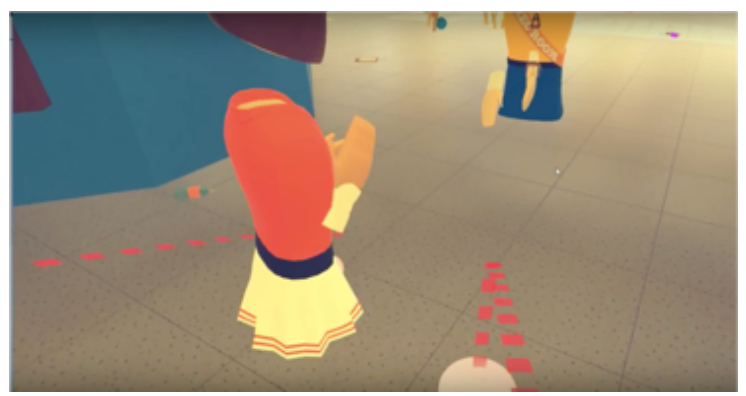

Figure 5: Prohibited teleportation in Rec Room 


\subsubsection{Onboarding as Social Buffering}

All of the interviews from open world social VR environments described effective onboarding as crucial for supporting pro-social interactions in social VR. Nearly all of our interviewees emphasized the important role that onboarding plays in providing new users with a sense of comfort with the basic embodied controls and interaction capabilities. This process typically occurs in a private room or area where new users can acclimate first before they enter a public environment. For Rec Room, this private area is explicitly designated as a "dorm room" with a mirror that affords "self-reflective properties sort of like being in a safe practice zone." In this way, onboarding provides a soft landing for users to prepare to interact with others.

\subsubsection{Spatial and Temporal Buffers}

This idea of a soft landing also applies to instances where a user is spawned into a new public environment. Several mentioned that it is important not to spawn users in the center of public environments. Instead it is better to spawn at entryways or on the edges of any defined architecture, so that users entering a new space have the opportunity to acclimate before encountering others. For Anyland, this social buffer is created through a time-delay. Experienced users are notified that new users have joined and can greet them, but this process only begins after a delay.

\subsection{Social VR with People You Already Know}

Unlike the open-world environments like Altspace VR, Anyland, VRChat, High Fidelity, and Rec Room, two of the applications we examined, Facebook Spaces and Mozilla Hubs were both closed environments intended to be utilized by people who already know one another.

Hubs software engineer, Greg Fodor, noted that the social VR ecosystem in general has tended to have a design bias towards encounters with strangers. This has meant that onboarding tends to focus on private (safe) environments as opposed to assuming that onboarding happens as a social experience with one friend or colleague guiding another. Likewise, the social VR design ecosystem tends to privilege aspects like blocking, muting, reporting and other moderation features.

Instead, Fodor explained that applications like Mozilla Hubs and Facebook Spaces emphasize a different set of pro-social features. These include:

(1) Porosity of media, insofar as images and 3D models from the web can easily be searched and brought into Hubs [Fig. 6]).
(2) Device interoperability and asymmetric participation, so that for example people engaging from a desktop environment have different "superpowers" than someone engaging with an HMD.

(3) Bridging VR and the outside world, as in the case of Facebook Spaces where users can broadcast live to their Newsfeed or call friends via Messenger.

To elaborate on category (3) above, Facebook Spaces emphasizes bridging VR with the "real world" of one's Facebook identity, for instance privileging default avatars generated from Facebook photos [Fig. 7]. By contrast, Mozilla Hubs focuses more on bringing the improvisational spirit and universal adaptability of the web into social VR.

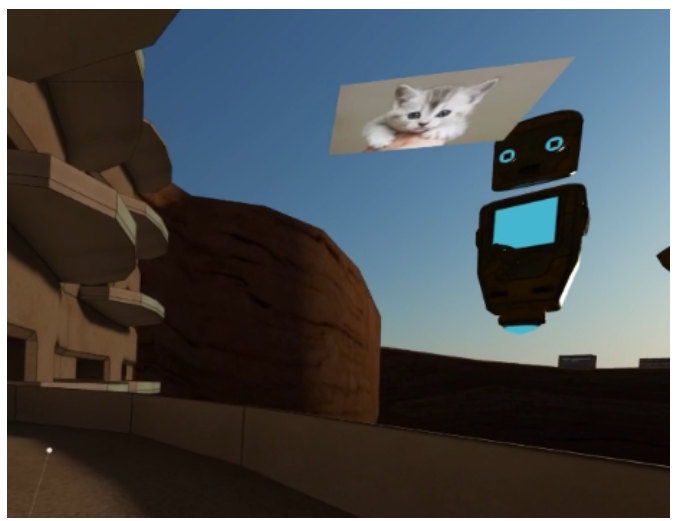

Figure 6: Mozilla Hubs user holding an image of a cat that was imported by a desktop participant

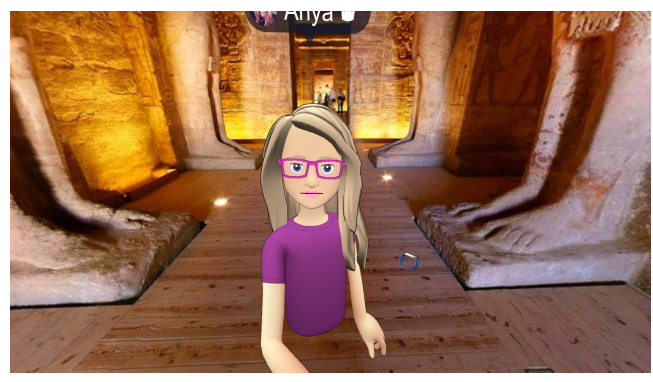

Figure 7: Photo-generated avatar in Facebook Spaces

\subsubsection{Learning from Others as Key to Onboarding}

Several of our interviewees emphasized the role that learning from others can play during onboarding. Anyland for example relies a lot on peer learning, as do Facebook Spaces and Mozilla Hubs. However, for closed environments like Facebook Spaces and Mozilla Hubs, social learning seems to supplant onboarding in significant ways. Mozilla's Fodor observed that "our entry flow from a design standpoint ... embraces the fact that [users] will be able to potentially coordinate that entry with someone else who already knows how things work, or who already has 
some prior experience.... We've prioritized the idea that you get in here typically with people you have some prior connection" And since these people gave you the link to a Hub, then they "by definition already [have] some prior experience, and [can] be your teacher." Likewise for Facebook Spaces, some new users are introduced to the application through the traditional 2D Facebook environment, when their friends broadcast Facebook Spaces through Facebook Live or call a friend via Messenger. In this way, aspects of onboarding may occur before a new user even owns a headset.

\section{DISCUSSION}

The interviews resulted in a list of key design topic areas for social VR designers to consider. While this list is likely not exhaustive, it represents a high level summation of the input from our various experts adapted into the language of design considerations. We note, however, that the relative priority of these categories depends upon the high level goals of the social VR creators, in particular whether one is designing an open world where strangers can meet or a private gathering space for friends from the physical world to connect in VR.

\section{Key Design Considerations and Opportunities:}

1. Leverage the known world to shape behavioral expectations and cue familiar social contexts through the aesthetics of place and architecture.

2. Position social catalysts and other attention focusing objects in the environment to serve as social lubricant.

3. Take advantage of social contagion (for example, through incentive structures, default expressive modalities for avatars, and cultural seeding practices associated with community outreach, and even nuances of language.)

4. Especially for open worlds, craft a safe onboarding experience that is separated from any public lobby area.

5. If human moderators are available, be proactive with community shaping in addition to reactive strategies of more traditional moderation.

6. Especially for moderator light environments, empower users to police/moderate their own user-created areas and activities.

7. Especially for closed social VR experiences with existing friends, assume peer learning is a key aspect of your onboarding flow.

8. Especially for closed social VR experiences with existing friends, consider the ways that your experience will bridge between VR and the outside world.
9. Especially for closed social VR experiences with existing friends, place priority on device interoperability, so that those who are novices can join without high end equipment. And consider asymmetrical participation models when people on different devices are engaging with one another.

Thus far we have called attention to design considerations in a number of areas. While the topics we have discussed may in some cases seem like reprisals of social VR's historic antecedents, there are significant differences in the ways that these topics play out in social VR which are worth elaborating here.

For instance, as described earlier, safe onboarding and spawning helps mitigate the abrupt sense of immersion among strangers, which can be physiologically overwhelming. While aspects of onboarding may be generally applicable in contexts like MMOs, social VR uniquely combines the experience of immersive remote presence with the uncanniness of interacting with (oddly embodied) strangers. While encounters with strangers are also a familiar feature of traditional MMOs, encountering strangers through an HMD is not. Moreover, to the best of our knowledge, HMD-based CVE research from the previous decades did not involve encounters with strangers in open worlds.

The connections that interview respondents made between environmental cues and social expectations also resonate with longstanding interests within HCI concerning the relationship between space (as a designed medium) and place (as the social fabric) [13, 15]. While features of place and space have been used to shape social expectations in traditional screen-based MMOs and immersive CVE research from previous decades, the context of social VR offers a new set of factors to consider. Particularly for openworld social VR, encountering unfamiliar strangers through remote presence accentuates the physiological experience of vulnerability when trying out a new activity. Consequently, commercial social VR heightens the anchoring role that place plays as a contextual scaffold for social interactions and activities. Familiar places like dance clubs, beaches, and rec centers activate social expectations and behavior patterns, ameliorate the weirdness and vulnerability of social VR, and provide coherent answers to the question "what am I supposed to be doing here?" Likewise, the geometry of circular tables and campfire gatherings ensure that shared eyelines and conversational proxemics can be accommodated in familiar ways.

Moreover, in social VR, traditional distinctions between place and space blur due to the complex ways that designers harness both the sociality of place and the 
affordances of space to elicit particular behaviors and social norms. The role played by social catalysts like ping pong in Rec Room or architectural features like the campfire in Altspace demonstrate the subtle ways that the geometry of space and cultural expectations of place are difficult to disentangle. The specificity of embodiment in social VR is key, here, as the affordances of shared eyelines and conversational orientation (or 'F-formations' [21, 25]) can only operate when they activate shared cultural understandings with others.

Like their predecessors from CVEs and Hybrid Media Spaces research of decades earlier [15], contemporary users of social VR also blur the boundaries between designerly practice and use, as they tinker with the embodied affordances of the medium. This capacity for users to take on the mindset of designers is evident in the capacity to create new worlds, customize avatars, playtest new games, invent new rituals, and experiment with new ways of bridging VR with the outside world.

Community management is similarly well-trodden phenomena in digital contexts. But features of embodiment in VR add fascinating new wrinkles to these sorts of practices. For example, town hall events in AltspaceVR leverage new embodied affordances such as giant emojis that emanate from audience members' bodies (in lieu of signals like clapping or hand raising). And in Rec Room, Q $\&$ A events utilize a giant bear (NPC) that sits on the stage stares in the direction of a roving microphone, so that attendees can track who is speaking at a given time.

Such examples seem to follow a similar pattern: (1) Designers leverage known context cues-such as familiar places-to provide an initial social scaffolding. (2) They rely on users' knowledge of social templates to communicate norms and action capacities in the environment. (3) They further shape action by calibrating spatial and embodied affordances or constraints. (4) They then pivot by stretching social expectations, sometimes in profoundly divergent ways (such as the giant bear example above). (5) But ultimately it is up to users to discover or perform this defamiliarizing move-from the known to the unknownfor example by inventing new kinds of embodied rituals like "head patting."

Aspects of peer learning and asymmetric participation in contemporary social VR also introduce new research questions. Users of Facebook Spaces teach new users about the platform by broadcasting themselves into their friends' Newsfeeds via Facebook Live or calling their friends via Messenger. Participants who watch this VR encounter on a
2D screen can post comments which appear as giant signposts in the Facebook Spaces environment. These forms of hybrid interaction raise questions about how learning occurs for those not in VR. Likewise, the emphasis on device interoperability in Mozilla Hubs means that different users can participate with complimentary asymmetric "superpowers." A participant on a desktop is able to search for relevant media and "drop" it into Hubs while those with HMDs are able to manipulate the media in ways that the desktop user cannot. These forms of asymmetric participation raise questions about how designers can best support interactions in virtual environments between collections of users who operate a range of devices and occupy disparate social contexts.

\section{CONCLUSIONS}

In this paper, we have engaged in an interview-driven study of design approaches gathered from industry experts to arrive at an emerging design framework for pro-social interaction in social VR. This framework is preliminary, but nevertheless suggests new avenues for empirical user research. For example, interviews with users could serve to validate, challenge, or otherwise further contextualize the design considerations identified as crucial for pro-social interaction in VR. For example, do users experience the aesthetics of place as a contextual anchor and social scaffolding the way that designers anticipate? How do social catalysts and particular architectural features structure embodied interaction for users in social VR contexts? And how should designers react to the rapidly growing expectations of agency of users who expect to design their own social contexts through in-world creation tools? What are the best ways to approach community engagement, moderation, and incentive structures, and what are the trade-offs of emphasizing one approach over another? As social mechanics and new communicative modalities become more familiar to users in VR, will such mechanics become standardized across the social VR ecosystem or will disparate models co-exist? And if so, what will be the stakes of these differences for users? Researchers have an opportunity to address these questions now, while the landscape is still evolving. In the future, social VR platforms may have the kind of broad impact on society that contemporary $2 \mathrm{D}$ social media currently maintain. This suggests a strong imperative for research communities to better understand the role that design choices play in this medium and to encourage thoughtfulness about the kind of social experiences we would like to nurture. 


\section{ACKNOWLEDGEMENTS}

We thank our interviewees for their generous contribution of time and expertise. This research was sponsored by Mozilla, and we extend special thanks to Lars Bergstrom, Jofish Kaye, Blair McIntyre, Miriam Avery, Greg Fodor, Jim Conrad, and the rest of the Mixed Reality team at Mozilla.

\section{REFERENCES}

[1] Benford, S. et al. 2001. Collaborative virtual environments. Communications of the ACM. 44, 7 (2001), 79-85.

[2] Benford, S. et al. 1995. User Embodiment in Collaborative Virtual Environments. Proceedings of the International Conference on Human Factors in Computing Systems (CHI'95). (1995), 242-249.

[3] Blascovich, J. 2002. A theoretical model of social influence for increasing the utility of collaborative virtual environments. Proceedings of the 4th International Conference on Collaborative Virtual Environments. (2002).

[4] Boellstorff, T. 2008. Coming of Age in Second Life. An Anthropologist Explores the Virtually Human.

[5] Boellstorff, T. et al. 2012. Ethnography and virtual worlds: A handbook of method. Princeton University Press.

[6] Bowers, J. et al. 1996. Talk and embodiment in collaborative virtual environments. Proceedings of the SIGCHI conference on Human factors in computing systems - CHI '96. (1996).

[7] Castronova, E. 2008. Exodus to the virtual world: how online fun is changing reality. Palgrave Macmillan.

[8] Castronova, E. 2005. Synthetic worlds: the business and culture of online games. University of Chicago Press.

[9] Churchill, E.F. and Snowdon, D. 1998. Collaborative virtual environments: An introductory review of issues and systems. Virtual Reality. (1998).

[10] Designing Facebook Spaces (Part 4) - Creating a VR interface: 2017. https://medium.com/@christauziet/designing-facebook-spaces-part-4creating-a-vr-interface-821861159495.

[11] Desurvire, H. and Kreminski, M. 2018. Are Game Design and User Research Guidelines Specific to Virtual Reality Effective in Creating a More Optimal Player Experience? Yes, VR PLAY. Design, User Experience, and Usability: Theory and Practice (Cham, 2018), 40-59.

[12] Dibbell, J. 1998. My tiny life: crime and passion in a virtual world. Holt.

[13] Dourish, P. 2006. Re-Space-ing Place: "Place " and "Space " Ten Years On. Computing. (2006).

[14] Doyle, D. 2010. Phenomenologies of practice: Making sense of virtual worlds and user-driven innovation. Making Sense of Virtual Worlds and User Driven Innovation (2010).

[15] Harrison, S. and Dourish, P. 1996. Re-Place-ing Space: The Roles of Place and Space in Collaborative Systems. 1996.

[16] He, Z. et al. 2017. PhyShare. Adjunct Publication of the 30th Annual ACM Symposium on User Interface Software and Technology - UIST '17 (New York, New York, USA, 2017), 17-19.

[17] Immersive Design: The Next 10 Years of Interfaces: 2018 https://uxdesign.cc/immersive-design-the-next-10-years-of-interfaces16122cb6eae6. Accessed: 2018-08-06.

[18] Isbister, K. et al. 2010. Designing games for learning. Proceedings of the 28th international conference on Human factors in computing systems - CHI ' 10 (New York, New York, USA, 2010), 2041.

[19] Isbister, K. 2010. Enabling Co-Located Physical Social Play: A Framework for Design and Evaluation. Game User Experience
Evaluation

[20] Isbister, K. and Mueller, F. "Floyd" 2015. Guidelines for the Design of Movement-Based Games and Their Relevance to HCI. HumanComputer Interaction. 30, 3-4 (May 2015), 366-399.

[21] Kendon, A. 1990. Conducting Interaction: Patterns of Behavior in Focused Encounters. Cambridge University Press, 1990.

[22] Ludlow, P. and Wallace, M. 2009. The Second Life Herald: The Virtual Tabloid that Witnessed the Dawn of the Metaverse. The MIT Press.

[23] Malaby, T. 2009. Making Virtual Worlds: Linden Lab and Second Life. Comstock Pub Assoc.

[24] Marquardt, N. and Greenberg, S. 2015. Proxemic Interactions: From Theory to Practice.

[25] Marshall, P. et al. 2011. Using F-formations to analyse spatial patterns of interaction in physical environments. Proceedings of the ACM 2011 conference on Computer supported cooperative work CSCW' 11 (New York, New York, USA, 2011), 445.

[26] McVeigh-Schultz, J. et al. 2018. What's It Mean to "Be Social" in VR? Proceedings of the 19th International ACM SIGACCESS Conference on Computers and Accessibility - DIS '18 (New York, New York, USA, 2018), 289-294.

[27] My first virtual reality groping: 2016. https://mic.com/articles/157415/my-first-virtual-reality-groping-sexualassault-in-vr-harassment-in-tech-jordan-belamire\#.7ECfifxnW.

[28] Ondrejka, C.R. 2004. A Piece of Place: Modeling the Digital on the Real in Second Life. SSRN Electronic fournal. (2004).

[29] Pallay, C. et al. 2009. Getting acquainted in Second Life. Proceedings of the International Conference on Advances in Computer Enterntainment Technology - ACE '09 (New York, New York, USA, 2009), 36.

[30] Pearce, C. 2009. Communities of Play: Emergent Cultures in Multiplayer Games and Virtual Worlds. The MIT Press.

[31] Perry, T.S. 2016. Virtual reality goes social. IEEE Spectrum. (2016).

[32] Saldaña, J. 2016. The Coding Manual for Qualitative Researchers. Sage Publications.

[33] Schild, J. et al. 2018. Applying Multi-User Virtual Reality to Collaborative Medical Training. IEEE Virtual Reality. March (2018).

[34] Schubert, T. et al. 1999. Embodied Presence in Virtual Environments. Visual Representations and Interpretations.

[35] Shriram, K. et al. 2017. All Are Welcome: Using VR Ethnography to Explore Harassment Behavior In Immersive Social Virtual Reality. Virtual Reality (Los Angeles, CA, 2017).

[36] Snowdon, D. et al. 2001. Collaborative Virtual Environments: Digital Places and Spaces for Interaction.

[37] Snowdon, D. et al. 2000. Collaborative Virtual Environments: Digital Spaces and Places for CSCW. Collaborative Virtual Environments. (2000).

[38] Sra, M. et al. 2018. Novel Interaction Techniques for Collaboration in VR. Extended Abstracts of the 2018 CHI Conference on Human Factors in Computing Systems - CHI '18 (New York, New York, USA, 2018), 1-8.

[39] Sra, M. et al. 2018. Your Place and Mine. Proceedings of the 2018 on Designing Interactive Systems Conference 2018 - DIS '18 (New York, New York, USA, 2018), 85-97.

[40] Taylor, T.L. 2006. Play Between Worlds: Exploring Online Game Culture.

[41] Thompson, M. et al. Rules, Roles , and Resources: Strategies to Promote Collaboration in Virtual Reality Contexts. Workshop Position Paper for CHI 2018.

[42] Wilcox, L.M. et al. 2006. Personal Space in Virtual Reality. ACM Transactions in Applied Perception. (2006). 